\title{
Frecuencia de agentes bacterianos asociados a mortalidad en cuyes de centros de crianza familiar-comercial en Canchis, Cusco
}

\author{
Frequency of bacterial agents associated with mortality in guinea pigs from \\ commercial-family breeding centres in Canchis, Cusco
}

\author{
José M. Angulo-Tisoc ${ }^{1,3}$, Luis M. Jara ${ }^{2}$, Joel I. Pacheco', Danilo Pezo'
}

\section{Resumen}

\begin{abstract}
El objetivo del presente estudio fue identificar los agentes bacterianos aislados y evaluar su susceptibilidad antibiótica a partir de casos de mortalidad en cuyes procedentes de dos centros de crianza familiar-comercial de la provincia de Canchis, Cusco. Se procesaron 230 animales (130 con lesiones respiratorias y 100 con lesiones enterohepáticas), tomándose muestras de hígado, pulmón, bazo e intestino. Se realizó un cultivo microbiológico y antibiograma en agar con discos de los principales antibióticos usados en sistemas de crianza de cuyes. A nivel de lesiones respiratorias se identificó a Streptococcus sp (47.1\%), Bordetella sp (26.4\%), Salmonella sp (13.8\%), Klebsiella $\mathrm{sp}$ (9.2\%) y Pasteurella sp (3.4\%). Asimismo, en cuyes con lesiones entéricas se identificó a Salmonella sp (56.8\%), E. coli (29.7\%), Citrobacter sp (7.6\%) y Klebsiella sp (5.9\%). La mayor frecuencia de aislados bibacterianos en cuadros respiratorios fue para Streptococcus sp y Bordetella sp, en tanto que para lesiones hepático-entéricas fue Salmonella sp y E. coli. La frecuencia general de resistencia en las cepas aisladas fue de 5.4 y $3.6 \%$ para enrofloxacina y trimetoprima-sulfametoxazol, respectivamente. Los resultados evidencian la variedad de géneros bacterianos presentes en procesos respiratorios y entérico-hepáticos en cuyes, así como una frecuencia baja de resistencia antibiótica en las cepas aisladas.
\end{abstract}

Palabra clave: antibiograma, cuy, bacterias, diarrea, neumonía, septicemia

\footnotetext{
${ }^{1}$ Grupo de Investigación en Producción y Sanidad de Ganadería Altoandina, Centro de Investigación IVITA - Maranganí, Universidad Nacional Mayor de San Marcos, Cusco, Perú

${ }^{2}$ Facultad de Medicina Veterinaria y Zootecnia, Universidad Peruana Cayetano Heredia, Lima, Perú

${ }^{3}$ Email: jangulot@unmsm.edu.pe; https://orcid.org/0000-0003-3238-5462
}

Recibido: 26 de octubre de 2020

Aceptado para publicación: 10 de abril de 2021

Publicado: 23 de junio de 2021

CLos autores. Este artículo es publicado por la Rev Inv Vet Perú de la Facultad de Medicina Veterinaria, Universidad Nacional Mayor de San Marcos. Este es un artículo de acceso abierto, distribuido bajo los términos de la licencia Creative Commons Atribución 4.0 Internacional (CC BY 4.0) [https:// creativecommons.org/licenses/by/4.0/deed.es] que permite el uso, distribución y reproducción en cualquier medio, siempre que la obra original sea debidamente citada de su fuente original 
The aim of this study was to identify bacterial agents isolated from fatal cases in guinea pigs as well as to evaluate their antibiotic susceptibility from two commercialfamily breeding centres in the province of Canchis, Cusco-Peru. In total, 230 animals (130 with respiratory lesions and 100 with enterohepatic lesions) were processed, including samples of the liver, lung, spleen and intestine. A microbiological culture and antibiogram were performed on agar with discs of the main antibiotics used in guinea pigs rearing. In the respiratory lesions, Streptococcus sp (47.1\%), Bordetella sp (26.4\%), Salmonella sp (13.8\%), Klebsiella sp (9.2\%) and Pasteurella sp (3.4\%) were identified. Likewise, in guinea pigs with enteric lesions, Salmonella sp (56.8\%), E. coli (29.7\%), Citrobacter sp $(7.6 \%)$ and Klebsiella sp (5.9\%) were identified. The highest frequency of bi-bacterial isolates in respiratory lesions was for Streptococcus sp and Bordetella sp, while for liver-enteric lesions it was Salmonella sp and E. coli was registered. The general frequency of resistance in the isolates was 5.4 and $3.6 \%$ for enrofloxacin and trimethoprimsulfamethoxazole, respectively. The results showed a variety of bacterial genera present in respiratory and enteric-hepatic processes in guinea pigs, as well as a low frequency of antibiotic resistance in the isolated strains.

Key words: antibiogram, guinea pig, bacteria, diarrhoea, pneumonia, septicaemia

\section{INTRODUCCIÓN}

La producción de cuyes constituye una actividad económica importante en varios países de la región Andina; sin embargo, las deficiencias sanitarias son una dificultad constante en el manejo y bioseguridad de las granjas. Existen diversos factores que predisponen a la presentación de enfermedades infecciosas bacterianas como son las deficientes condiciones higiénicas y sanitarias, cambios bruscos medioambientales, alta densidad animal y déficit nutricional, entre otros (Chauca, 1997; Avilés et al., 2014; SánchezMacías et al., 2018).

Streptococcus pneumoniae es una bacteria relacionada a problemas respiratorios (van der Linden et al., 2009; Harkness et al., 2010), reportándose en un $8.8 \%$ de procesos infecciosos en crianzas intensivas de cuyes en Lima (Killerby et al., 2019). La transmisión se da por aerosoles, contacto directo o verticalmente al nacimiento. Los animales pueden ser portadores subclínicos o presen- tar signos clínicos como anorexia, secreción nasal u ocular, estornudos, tos, disnea y problemas reproductivos (Shomer et al., 2015). Por otro lado, dentro de las principales lesiones observadas se describen la pleuritis fibrinopurulenta, pericarditis, peritonitis y neumonía supurativa (Percy y Barthold, 2007; Harkness et al., 2010).

Bordetella sp se encuentra también asociada a afecciones respiratorias (Fox et al., 2002). La infección puede manifestarse con inapetencia, depresión, flujo respiratorio superior, disnea, cianosis o afecciones reproductivas (Brabb et al., 2012; Shomer et al., 2015). Junto con Pasteurella sp son comensales de la mucosa orofaríngea y tracto gastrointestinal (Songer y Post, 2005), reportada en crianzas familiar-comercial a partir de cuyes con diversa signología (Morales, 2017).

Salmonella sp es un agente transmitido principalmente vía fecal-oral que genera daño entérico en cuyes. Salmonella enterica subsp. enterica serovar Typhimurium $(S$. 
Typhimurium) es la serovariedad más frecuentemente aislada en animales sanos portadores (Harkness et al., 2010; Chero et al., 2017). En Perú se reportan aislamientos superiores al $86 \%$ a partir de lesiones en hígado y bazo, y en menor porcentaje a nivel de pulmón, útero, vesícula biliar o intestino (Matsuura et al., 2010; Layme et al., 2011).

Otra bacteria como E. coli genera una signología variable con efectos adversos en la barrera intestinal (Harkness et al., 2010; Brabb, 2012; Ren et al., 2017). La transmisión se genera producto de las deficientes condiciones sanitarias y factores ambientales estresantes, ocasionando disbiosis y mortalidad (O'Rourke, 2004). En cuyes lactantes y reproductores ha sido aislada entre 23 y $40 \%$ en casos de índole infecciosa (Chuquizuta y Morales, 2017; Obregón et al., 2018; Killerby et al., 2019). Citrobacter sp es además comúnmente identificada en cuyes reproductores de crianza familiar-comercial (Morales, 2012; Killerby et al., 2019); además, es uno de los agentes implicados en cuadros de mortalidad en cuyes neonatos a partir de animales afectados (Chuquizuta y Morales, 2017; Obregón et al., 2018). A su vez, el agente oportunista Klebsiella pneumoniae genera afecciones respiratorias, cuadros septicémicos agudos, y lesiones a nivel toráxico y abdominal (Merino et al., 1992; Percy y Barthold, 2007; Shomer et al., 2015).

No se dispone de muchos estudios de aislados bacterianos y su susceptibilidad antibiótica en cuyes, a excepción de Salmonella (Matsuura et al., 2010; Salvatierra et al., 2018; Huamán et al., 2020). El estudio de la susceptibilidad antibiótica in vitro brinda información complementaria para el abordaje terapéutico, lo cual permite adecuar tratamientos dirigidos que sean efectivos y a su vez no generen resistencia en el tiempo (Cantón, 2010).

La región del Cusco es una zona altamente productiva de cuyes, siendo la provincia de Canchis una de las más importantes
(INEI, 2012); sin embargo, son escasos los estudios sobre identificación y frecuencia de patógenos bacterianos asociados a problemas respiratorios y entéricos. El objetivo del presente estudio fue determinar la frecuencia de patógenos bacterianos asociados a lesiones respiratorias y enterohepáticas, así como evaluar el perfil de susceptibilidad antimicrobiana de los aislados provenientes de centros de crianza familiar-comercial de cuyes en Canchis, Cusco.

\section{Materiales y Métodos}

\section{Población de Estudio}

Se analizaron 230 cuyes de tres etapas productivas: gazapo $(n=69)$, recría $(n=74)$ y adulto $(\mathrm{n}=87)$, tanto machos $(\mathrm{n}=107)$ como hembras $(\mathrm{n}=123)$. Los animales presentaban lesiones macroscópicas a nivel de cavidad toráxica como neumonía, pericarditis, neumotórax $(\mathrm{n}=100)$ y a nivel entérico-hepático como ascitis, abscesos, congestión, focos necróticos o hemorrágicos, incluyendo bazo y nódulos linfáticos mesentéricos $(\mathrm{n}=130)$. Los especímenes tuvieron desde un día de nacidos hasta 14 meses de edad. Fueron remitidos antes de las dos horas después del sacrificio al Laboratorio de Bacteriología de la estación experimental del Centro de Investigaciones IVITA-UNMSM, sede Maranganí, Cusco, entre septiembre de 2018 a marzo de 2019. Los animales fueron sacrificados por los mismos productores, propietarios de dos centros de crianza familiar-comercial de la provincia de Canchis, Cusco.

\section{Diagnóstico Microbiológico}

La toma de muestra de los órganos (hígado, bazo, pulmón e intestino) se realizó durante la necropsia. El cultivo de las muestras se hizo mediante siembra microbiológica directa por agotamiento en agares McConkey, TSA (tripticasa de soya) y sangre (tripticasa de soya base con $5 \%$ de sangre desfibrinada de carnero). Las placas fueron puestas en 
anaerobiosis dentro de una jarra de Brewer hermética utilizando el sistema Anaerocult ${ }^{\circledR}$ $\mathrm{C}$, e incubadas por $24-48 \mathrm{~h}$ a $37^{\circ} \mathrm{C}$. Las colonias resultantes fueron identificadas según sus características y morfología macroscópica, tipo de hemólisis, tinción de Gram, actividad catalasa y pruebas bioquímicas para el caso de Gram negativas (Vadillo et al., 2002).

\section{Susceptibilidad Antibiótica in vitro}

La evaluación de la susceptibilidad antibiótica se realizó de acuerdo con el Manual de Procedimientos para la Prueba de Sensibilidad Antimicrobiana por el Método de Disco Difusión (INS, 2002). Para esto, se tomaron varias colonias de un cultivo bacteriano puro y se suspendieron en agua destilada estéril a una concentración equivalente a 0.5 de la escala de McFarland. La suspensión se sembró en tres direcciones con un hisopo estéril sobre una placa con agar Mueller-Hinton, se dejó reposar cinco minutos a temperatura ambiente y se colocó un máximo de cinco discos de antibióticos sobre la superficie del agar. Se incubó seguidamente a $37^{\circ} \mathrm{C}$ por 24 horas.

Los discos comerciales de antibióticos correspondieron a los utilizados en el manejo sanitario de cuyes: enrofloxacina $(5 \mu \mathrm{g})$, ciprofloxacina $(5 \mu \mathrm{g})$, gentamicina $(10 \mu \mathrm{g})$, trimetoprima-sulfametoxazol $(1.25 / 23.75 \mu \mathrm{g})$, oxitetraciclina $(30 \mu \mathrm{g})$ y fosfomicina $(20 \mu \mathrm{g})$ (Donnelly, 2020). Los halos de inhibición fueron medidos con una regla y expresados en milímetros (mm). Se determinó la cepa como resistente, susceptible o intermedio según indicaciones del fabricante (Oxoid, Inglaterra).

\section{Análisis de Resultados}

Los resultados se expresaron en cuadros de frecuencias con estadística descriptiva, según el tipo de patógeno aislado, clase de antibiótico evaluado y distribución de aislados con base al sexo y etapa productiva de los animales.

\section{Resultados}

En $87 \%(87 / 100)$ de los cuyes con lesiones respiratorias se aisló al menos un tipo de agente bacteriano, resultando 124 cepas aisladas. Asimismo, en 90.8\% (118/130) de los cuyes con lesiones enterohepáticas se aisló al menos un agente bacteriano resultando 153 cepas aisladas (Cuadro 1).

La frecuencia de aislamientos con dos agentes bacterianos resultó principalmente para Streptococcus sp y Bordetella sp con $52.9 \%$ (46/87), Streptococcus sp y Salmonella sp con 33.3\% (29/87) y Bordetella $\mathrm{sp}$ y Salmonella sp con $13.8 \%(12 / 87)$ a partir de lesiones respiratorias, y Salmonella sp y E. coli con $36.4 \%(43 / 118)$ a partir de lesiones enterohepáticas.

En la distribución de animales de donde se aislaron agentes bacterianos respiratorios según la etapa productiva, $26.5 \%(23 / 87) \mathrm{de}$ gazapos y $16.1 \%(14 / 87)$ de recrías resultaron positivos a Streptococcus sp; además, $13.8 \%(12 / 87)$ de recrías y $10.3 \%(9 / 87) \mathrm{de}$ gazapos a Bordetella sp (Figura 1A). Con relación al sexo, 52.9\% (46/87) de machos resultaron con lesiones respiratorias y $51.7 \%$ $(61 / 118)$ de hembras resultaron con lesiones enterohepáticas. Respecto a las lesiones enterohepáticas, $36.4 \%(43 / 118)$ de recrías y $15.3 \%(18 / 118)$ de adultos resultaron positivos a Salmonella sp, además del 22.9\% (27/ $118)$ de recrías y $4.2 \%(5 / 118)$ de gazapos a E. coli (Figura 1B).

En cuanto a los resultados de la evaluación de susceptibilidad antibiótica, se observó una frecuencia de sensibilidad de más del $50 \%$ de cepas para todos los antibióticos evaluados, y frecuencias de resistencia de 5.4\% para enrofloxacina, $3.6 \%$ para trimetoprimasulfametoxazol y $0.7 \%$ para gentamicina. Asimismo, se obtuvo una frecuencia de susceptibilidad intermedia de 21.3 y $12.6 \%$ para enrofloxacina y trimetoprima-sulfametoxazol, respectivamente (Cuadro 2). 
Cuadro 1. Tipos de bacterias aisladas de cuyes con lesiones respiratorias y enterohepáticas de dos centros de crianza familiar-comercial de la provincia de Canchis, Cusco (2018-2019

\begin{tabular}{llcc}
\hline & Bacteria & \multicolumn{2}{c}{ Aislados } \\
\cline { 3 - 4 } & & $\mathrm{n}$ & $\%$ \\
\hline Lesiones respiratorias & Streptococcus $\mathrm{sp}$ & 41 & 47.1 \\
$(\mathrm{n}=89)$ & Bordetella $\mathrm{sp}$ & 23 & 26.4 \\
& Salmonella $\mathrm{sp}$ & 12 & 13.8 \\
& Klebsiella $\mathrm{sp}$ & 8 & 9.2 \\
& Pasteurella $\mathrm{sp}$ & 3 & 3.4 \\
Lesiones enterohepáticas & Salmonella $\mathrm{sp}$ & 67 & 56.8 \\
$(\mathrm{n}=118)$ & E. coli & 35 & 29.7 \\
& Citrobacter $\mathrm{sp}$ & 9 & 7.6 \\
& Klebsiella $\mathrm{sp}$ & 7 & 5.9 \\
\hline
\end{tabular}

Cuadro 2. Perfil del antibiograma de cepas bacterianas aisladas $(n=277)$ a partir de lesiones respiratorias y enterohepáticas en cuyes de dos centros de crianza familiar-comercial de la provincia de Canchis, Cusco (2018-2019

\begin{tabular}{lccc}
\hline \multirow{2}{*}{ Antibiótico } & \multicolumn{3}{c}{ Frecuencia (\%) de susceptibilidad antimicrobiana } \\
\cline { 2 - 4 } & Sensible & Intermedio & Resistente \\
\hline Enrofloxacina & 73.3 & 21.3 & 5.4 \\
Trimetoprima- & 83.8 & 12.6 & 3.6 \\
sulfametoxazol & 93.1 & 6.1 & 0.7 \\
Gentamicina & 96.0 & 4.0 & 0 \\
Ciprofloxacina & 95.3 & 4.7 & 0 \\
Oxitetraciclina & 93.9 & 6.1 & 0 \\
Fosfomicina & & & \\
\hline
\end{tabular}

La frecuencia de cepas resistentes fue mayor en E. coli $(12.5 \%)$ para enrofloxacina, seguido de Salmonella sp (7.3\%), mientras que la resistencia para Salmonella sp para trimetoprima-sulfametoxazol y gentamicina fue del 7.7 y $2.2 \%$, respectivamente (Cuadro 3). 
A

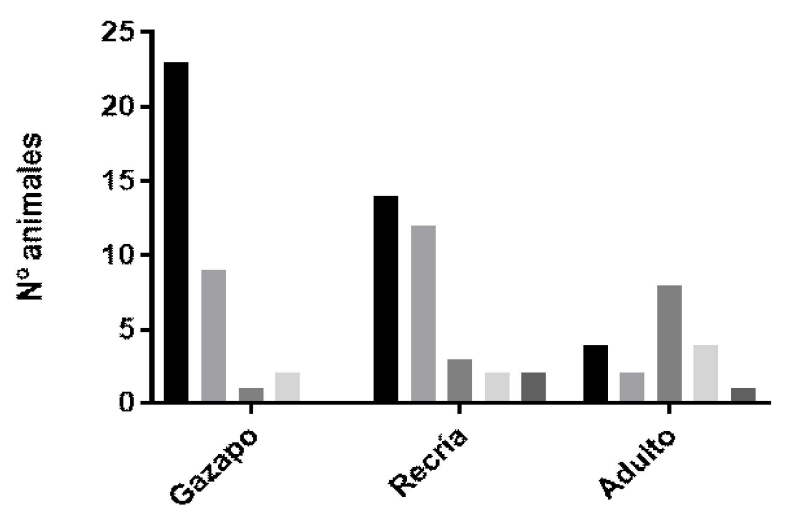

- Streptococcus sp.

Bordetella sp.

- Salmonella sp.

Klebsiella sp.

- Pasteurella sp.

B

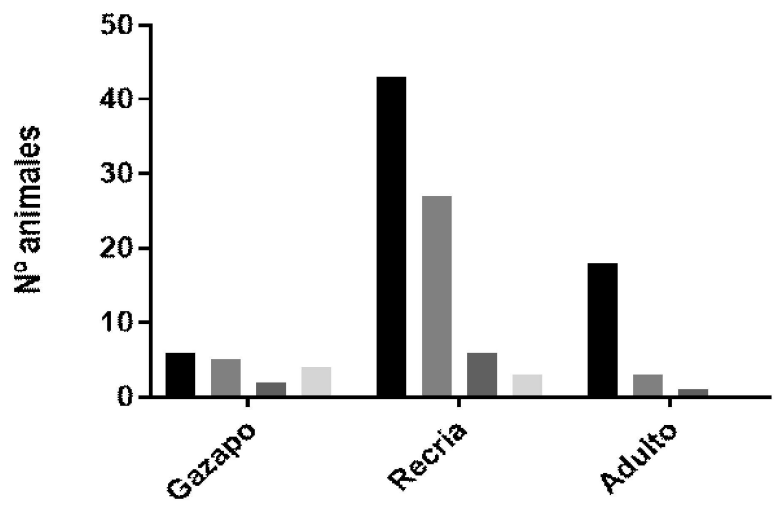

- Salmonella sp.

E. coli

- Citrobacter sp

Klebsiella sp.

Figura 1. Distribución de aislamientos bacterianos en cuyes con lesiones macroscópicas a nivel de órganos de cavidad toráxica $(n=87)$ y a nivel entérico-hepático $(n=118)$. A) Cuyes con lesiones en cavidad torácica y B) Con lesiones enterohepáticas, según la etapa productiva

Cuadro 3. Frecuencia de cepas resistentes según género bacteriano aislado y tipo de antibiótico evaluado

\begin{tabular}{lccc}
\hline \multirow{2}{*}{ Antibiótico } & \multicolumn{3}{c}{ Frecuencia de resistencia antimicrobiana } \\
\cline { 2 - 4 } & $\begin{array}{c}\text { Streptococcus } \mathrm{sp} \\
(\mathrm{n}=63)\end{array}$ & $\begin{array}{c}\text { Salmonella } \mathrm{sp} \\
(\mathrm{n}=91)\end{array}$ & $\begin{array}{c}\text { E. coli } \\
(\mathrm{n}=48)\end{array}$ \\
\hline Enrofloxacina & 1.58 & 7.28 & 12.5 \\
Trimetoprima-sulfametoxazol & 3.17 & 7.69 & 0.48 \\
Gentamicina & 0 & 2.19 & 0 \\
\hline
\end{tabular}




\section{Discusión}

En más del $80 \%$ de cuyes afectados en el presente estudio se aisló al menos un agente bacteriano, resultado similar al obtenido por Chuquizuta y Morales (2017) y Killerby et al. (2019) en gazapos y reproductoras a partir de especímenes muertos en sistemas de crianza comercial de Lima. Esto demuestra el alto porcentaje de casos infecciosos donde los agentes bacterianos podrían estar involucrados, además de otras consideraciones multifactoriales.

Streptococcus sp fue la bacteria aislada en mayor frecuencia a partir de lesiones respiratorias, seguido de Bordetella sp, la cual no presenta antecedentes de reporte en Perú para cuyes. Por otro lado, se encontró además Salmonella $\mathrm{sp}$, agente principalmente reportado en problemas entéricos, cuya presencia en localizaciones extra entéricas estaría vinculada a cuadros severos de septicemia (Layme et al., 2011). Además, se aisló en menor grado Klebsiella sp y Pasteurella $\mathrm{sp}$, las cuales también han sido reportadas en cuyes de Lima y Ancash en sistemas de crianza comercial y familiar-comercial, respectivamente (Chuquizuta y Morales, 2017; Morales, 2017; Obregón et al., 2018; Killerby et al., 2019).

Mientras que Salmonella sp fue la bacteria aislada en mayor frecuencia a partir de lesiones enterohepáticas, coincidiendo con otros estudios que registran su presencia en diversas etapas productivas (Matsuura et al., 2010; Obregón et al., 2018; Killerby et al., 2019). La genotipificación de cepas a partir de cuadros severos resultaría relevante, aunque el serovar mayormente reportado corresponde a $S$. Typhimurium, incluyendo aislamientos a partir de cuadros subclínicos (Harkness et al., 2010; Layme et al., 2011; Chero et al., 2017). El aislamiento de Klebsiella sp y Citrobacter sp es similar a otros estudios (Chuquizuta y Morales, 2017; Obregón et al., 2018; Killerby et al., 2019), lo cual evidencia la presencia ubicua de diferentes géneros de enterobacterias patógenas en cuyes.

Bacterias del género Streptococcus en cuyes ocasionan generalmente cuadros neumónicos y linfadenitis cervical, siendo $S$. pneumoniae y $S$. equi subsp. zooepidemicus las especies más frecuentemente asociadas (Percy y Barthold, 2007; Brabb et al., 2012). Las etapas productivas mayormente afectadas en el presente estudio fueron gazapos y recrías, lo cual podría verse asociado a la transmisión vertical o a diversos factores estresantes como inadecuada ventilación, cambios de temperatura, problemas nutricionales, entre otros, que pueden predisponer a la infección (Percy y Barthold, 2007; Shomer et al., 2015).

Referente al antibiograma obtenido para Salmonella, se encontró para enrofloxacina una frecuencia de susceptibilidad intermedia $(19 \%)$, siendo menor a lo reportado en cuyes de crianza familiar-comercial en Cajamarca y Moquegua (Caballero, 2015). Cabe destacar la baja resistencia encontrada en el presente estudio en comparación con otras investigaciones llevadas a cabo en los últimos cinco años en Cajamarca, Moquegua y Lima en crianzas familiar-comercial y comercial, donde incluso se ha evidenciado cepas multidrogo resistentes (Caballero, 2015; Salvatierra et al., 2018; Huamán et al., 2020).

Por otro lado, para trimetoprimasulfametoxazol se obtuvo una susceptibilidad intermedia de más del 10\%, diferente a reportes en Ancash, Cajamarca, Moquegua y Lima en crianzas familiar-comercial y comercial donde este fármaco presentó una sensibilidad mayor al 97\% (Matsuura et al., 2010; Caballero, 2015; Salvatierra et al., 2018). No obstante, otros estudios han registrado frecuencias de resistencias mayores a $67 \%$ en Cajamarca y Lima (Vásquez, 2019; Huamán et al., 2020). Dichas variaciones podrían encontrarse influenciadas principalmente por el uso inadecuado de antibióticos en cuanto a 
dosis, tiempo de tratamiento y diagnóstico incorrecto, entre otras causas. Es así que se requiere de estudios adicionales para obtener información sobre la percepción del uso correcto de antibióticos en las diferentes regiones del país.

Salmonella sp fue la bacteria que presentó una mayor frecuencia de resistencia, seguido de E. coli y Streptococcus sp a los antibióticos comúnmente utilizados como la enrofloxacina y trimetoprima-sulfametoxazol. Si bien las resistencias encontradas fueron bajas para antibióticos de amplio espectro, la susceptibilidad intermedia hallada podría potencialmente convertirse en resistente con el tiempo; por lo que es necesario realizar estudios de vigilancia antimicrobiana en el tiempo en diferentes localidades y sistemas de crianza.

$E$. coli se encuentra asociada a cuadros diarreicos en cuyes, siendo aislada en el presente estudio como el segundo agente implicado en lesiones enterohepáticas. Su alta carga se ve influenciada por la crianza en pozas, donde las heces se acumulan en el ambiente y puede existir un mayor riesgo de contaminación del alimento, forraje y agua, entre otros (Percy y Barthold, 2007; Harkness et al., 2010; Killerby et al., 2019). Asimismo, la mayor frecuencia fue a partir de recrías, donde se ha reportado desde diarreas transitorias leves hasta la muerte de camadas enteras (Richardson, 2000; Harkness et al., 2010). Si bien esta bacteria puede ser autolimitante en casos leves, los estudios de caracterización molecular son necesarios para identificar los patotipos diarrogénicos que podrían estar circulando en los sistemas de crianza.

Aunque no se identificó la especie de Citrobacter en el presente estudio, se ha reportado a $C$. freundii como una de las más comunes, además de $C$. diversus y $C$. amalonaticus en casos de diarrea (Ocholi et al., 1988; Chuquizuta y Morales, 2017). Asimismo, Klebsiella sp se aisló tanto de problemas entéricos como respiratorios, posiblemente influenciado por la implicancia como patógeno oportunista en animales inmunosuprimidos (Merino et al., 1992). Mientras que en Pasteurella sp, la especie más reportada es $P$. multocida en procesos infecciosos (Fox et al., 2002; Percy y Barthold, 2007).

No se pudieron obtener los antecedentes de los centros de crianza ni de los animales con signología previa al sacrificio; tampoco se pudo evaluar las lesiones a nivel histológico, lo que hubiera contribuido a la comprensión de la patogénesis y etiología. De otra parte, tampoco se llegó a identificar las especies de bacterias aisladas; sin embargo, por la casuística pueden relacionarse a las especies frecuentemente reportadas, lo que es relevante para conocer el estatus epidemiológico en galpones de cuyes. La alta frecuencia encontrada de enterobacterias como Salmonella sugiere realizar evaluaciones periódicas de la carga bacteriana ambiental en los sistemas de crianza, a fin de mitigar posibles brotes. Asimismo, las asociaciones de más de un tipo de bacteria podrían tener implicancia en cuadros infecciosos severos, por lo que es importante aislar e identificar todos los géneros bacterianos involucrados con la finalidad de establecer la antibioterapia de amplio espectro más adecuada.

\section{Conclusiones}

- Streptococcus sp y Bordetella sp fueron los agentes infecciosos mayormente aislados de cuyes con lesiones respiratorias, principalmente en gazapos y recrías de dos centros de crianza familiar-comercial de la provincia de Canchis, Cusco.

- Salmonella sp y E. coli fueron los agentes infecciosos mayormente aislados en animales con lesiones hepático-entéricas, principalmente en recrías.

- Los aislados bacterianos mostraron alta susceptibilidad antimicrobiana in vitro para la mayoría de los antibióticos utilizados en sistemas de crianza de cuyes. 
- Streptococcus sp y Salmonella sp mostraron bajos niveles de resistencia antimicrobiana y menos del $50 \%$ de susceptibilidad intermedia para enrofloxacina y trimetoprima-sulfametoxazol.

\section{Literatura Citada}

1. Avilés D, Martínez MA, Landi V, Delgado JV. 2014. El cuy (Cavia porcellus): un recurso andino de interés agroalimentario. Anim Genet Resour 55: 87-91. doi: 10.1017/S2078633614000368

2. Brabb T, Newsome D, Burich A, Hanes M. 2012. Infectious diseases. In: Suckow MA, Stevens KA, Wilson RP (eds). The laboratory rabbit, guinea pig, hamster, and other rodents. USA: Academic Press p 637 - 683.

3. Caballero R. 2015. Caracterización fenotípica y genotípica de salmonelosis en Cavia porcellus (cuyes) en las regiones de Cajamarca, Lima y Moquegua. Tesis de Médico Veterinario y Zootecnista. Lima: Univ. Peruana Cayetano Heredia. 48 p.

4. Cantón R. 2010. Lectura interpretada del antibiograma: una necesidad clínica. Enferm Infecc Microbiol Clin 28: 375385. doi: 10.1016/j.eimc.2010.01.001

5. Chauca L. 1997. Producción de cuyes (Cavia porcellus). Roma: Organización de las Naciones Unidas para la Agricultura y la Alimentación (FAO). 80 p.

6. Chero A, Rosadio R, Marcelo G, Diaz G, Jiménez R, Castro Y, Maturrano L. 2017. Identificación molecular de Salmonella Typhimurium en cuyes al primer parto mediante la técnica de PCR múltiple. Rev Inv Vet Perú 28: 679-686. doi: 10.15381/rivep.v28.i3.13288

7. Chuquizuta C, Morales S. 2017. Identificación de agentes bacterianos aislados de gazapos muertos de cuyes en una granja de crianza intensiva en Lima, Perú. REDVET 18(12). [Internet]. Disponible en: https://www.redalyc.org/pdf/ 636/63654640041.pdf
8. Donnelly TM. 2020. Guinea pigs. The Merck Veterinary Manual. [Internet]. Disponible en https://www.msdvetmanual.com/exotic-andlaboratoryanimals/rodents/guinea-pigs?query=guinea pigs

9. Fox JG, Anderson LC, Loew FM, Quimby $F W$. 2002. Laboratory animal medicine. New York, USA: Academic Press. 900 p.

10. Harkness JE, Turner PV, Van de Woude S, Wheler CL. 2010. Harkness and Wagner's biology and medicine of rabbits and rodents. $5^{\text {th }}$ ed. USA: Wiley Blackwell. $472 \mathrm{p}$

11. Huamán M, Pérez C, Rodríguez J, Killerby M, Lovón S, Chauca L. 2020. Caracterización genética y patrones de resistencia antimicrobiana en cepas de Salmonella enterica subsp. enterica serovar Typhimurium en cuyes de crianza intensiva. Rev Inv Vet Perú 31: e17542. doi: $10.15381 . v 31 i 1.17542$

12. Instituto Nacional de Estadística e Informática (INEI). 2012. IV Censo Nacional Agropecuario 2012. [Internet], [24 de Marzo 2015]. Disponible en: http:// censos.inei.gob.pe/Cenagro/redatam/\#

13. [INS] Instituto Nacional de Salud. 2002. Manual de procedimientos para la prueba de sensibilidad antimicrobiana por el método de disco difusión. Serie de Normas Técnicas N. ${ }^{\circ}$ 30. Lima, Perú. 67 p.

14. Killerby M, Huamán M, Chauca L. 2019. Identificación de los agentes bacterianos relacionados con mortalidad en cuyes reproductores de crianza intensiva. Salud Tecnol Vet 2: 9-16. doi: 10.20453/stv.v7i2.3676

15. Layme A, Perales $R$, Chavera A, Gavidia C, Calle S. 2011. Lesiones anatomopatológicas en cuyes (Cavia porcellus) con diagnóstico bacteriológico de Salmonella sp. Rev Inv Vet Perú 22:369-376. doi: 10.15381/rivep.v22i4.14513

16. Matsuura A, Morales $S$, Calle $S$, Ara $M . ~ 2010$. Susceptibilidad a antibacterianos in vitro de Salmonella enterica 
aislada de cuyes de crianza familiar-comercial en la provincia de Carhuaz, Áncash. Rev Inv Vet Perú 21: 93-99. doi: 10.15381/ rivep.v21i1.355

17. Merino S, Camprubí S, Albertí S, Benedí VJ, Tomás J. 1992. Mechanisms of Klebsiella pneumoniae resistance to complement - mediated killing. Infect Inmun 6: 2529-2535.

18. Morales S. 2012. Patógenos oportunistas por transmisión fecal-oral en cuyes reproductores introducidos al distrito de San Marcos. Científica 9: 33-38.

19. Morales S. 2017. Patógenos bacterianos y parasitarios más frecuentes en cuyes de crianza familiar-comercial en tres distritos de la provincia de Bolognesi, departamento de Ancash en época de seca. Tesis de Maestría. Lima, Perú: Univ. Nacional Mayor de San Marcos. 72 p.

20. Obregón R, Serrano-Martínez E, Chauca L. 2018. Causas de mortalidad neonatal en cobayos (Cavia porcellus) durante la estación fría en el Instituto Nacional de Innovación Agraria, Lima Perú. Salud Tecnol Vet 2: 93-99. doi: 10.20453/stv.v6i2.3463

21. Ocholi RA, Chima JC, Uche EM, Oyetunde IL. 1988. An epizootic infection of Citrobacter freundii in a guinea pig colony: short communication. Lab Animal 22: 335-336.

22. O'Rourke DP. 2004. Diseases and problems of guinea pigs. In: Quesenberry KE, Carpenter JW (eds). Ferrets, rabbits, and rodents: clinical medicine and surgery: includes sugar gliders and hedgehogs. $2^{\text {nd }}$ ed. St. Louis, USA: Saunders. p 245-254.

23. Percy D, Barthold S. 2007. Pathology of laboratory rodents and rabbits. $3^{\text {rd }} \mathrm{ed}$. Iopwa, USA: Blackwell Publishing. $325 \mathrm{p}$.

24. Ren $X, Z h u$ Y, Gamallat $Y, M a S$, Chiwala G, Meyiah A, Xin Y. 2017. E. coli $\mathrm{O} 124 \mathrm{~K} 72$ alters the intestinal barrier and the tight junctions proteins of guinea pig intestine. Biomed Pharmacother 94:
468-473. doi: 10.1016/j.biopha.2017.07.123 .

25. Richardson V. 2000. Diseases of domestic guinea pigs. $2^{\text {nd }}$ ed. Oxford: Blackwell Science, $144 \mathrm{p}$.

26. Salvatierra G, Rimac R, Chero A, Reyna I, Rosadio R, Maturrano $L$. 2018. Resistencia antimicrobiana y genotipificación de cepas de Salmonella typhimurium aisladas de cuyes $(\mathrm{Ca}$ via porcellus) provenientes de granjas de producción intensiva de la ciudad de Lima, Perú. Rev Inv Vet Perú 29: 319-327. doi: 10.15381/rivep.v29i1.14089

27. Sánchez-Macías D, Barba-Maggi L, Morales-delaNuez A, Palmay-Paredes J. 2018. Guinea pig for meat production: a systematic review of factors affecting the production, carcass and meat quality. Meat Sci 143: 165-176. doi: 10.1016/j.meatsci.2018.05.004.

28. Shomer NH, Holcombe H, Harkness JE. 2015. Biology and diseases of guinea pigs. In Fox JG, Anderson LC, Otto $\mathrm{GM}$, et al. (eds). Laboratory animal medicine. $3^{\text {rd }}$ ed. Oxford: Academic Press. p 247-283.

29. Songer JG, Post KW. 2005. Veterinary microbiology: bacterial and fungal agents of animal disease. St. Louis, USA: Elsevier Saunders. 448 p.

30. Vadillo S, Piriz S, Mateos E. 2002. Manual de microbiología veterinaria. España: McGraw-Hill Interamericana. 704 p.

31. van der Linden M, Al-Lahham A, Nicklas W, Reinert RR. 2009. Molecular characterization of pneumococcal isolates from pets and laboratory animals. Plos One 4: e8286. doi:10.1371/ journal.pone. 0008286

32. Vásquez H. 2019. Susceptibilidad antimicrobiana de Salmonella spp. aislada de cuyes de tres zonas productoras de la Región Cajamarca-2018. Tesis de Médico Veterinario. Cajamarca, Perú: Univ. Nacional de Cajamarca. 78 p. 